\title{
Staying safe with laparoscopic cholecystectomy: the use of landmarking and intraoperative time-outs
}

\author{
Shirley Xiaoxuan Deng ${ }^{1 \wedge}$, Alice Zhu ${ }^{1}$, Melanie Tsang ${ }^{1,2,3}$, Brittany Greene $^{1,3}$, Shiva Jayaraman ${ }^{1,2,3}$ \\ ${ }^{1}$ Temerty Faculty of Medicine, University of Toronto, Toronto, Canada; ${ }^{2}$ HPB Service, St. Joseph's Health Centre, Unity Health Toronto, Canada; \\ ${ }^{3}$ Division of General Surgery, University of Toronto, Toronto, Canada \\ Correspondence to: Shiva Jayaraman, MD, MESc, FRCSC FACS. St. Joseph's Health Centre, 1600 Bloor St W, Toronto, ON M6P 1A7, Canada. \\ Email: shiva.jayaraman@unityhealth.to.
}

Received: 21 January 2021; Accepted: 07 April 2021; Published: 14 May 2021.

doi: $10.21037 /$ aos-21-1

View this article at: http://dx.doi.org/10.21037/aos-21-1

\section{Introduction}

Laparoscopic cholecystectomy is one of the most common procedures in General Surgery. Devastating complications of laparoscopic cholecystectomy include vascular and bile duct injury. Bile duct injury is an especially serious and potentially life-threatening complication that occurs at a reported rate of $0.08-1.5 \%$ (1-4). Vasculobiliary injuries can be prevented by following an algorithmic approach to laparoscopic cholecystectomy. At our institution, we have devised a culture for safe laparoscopic cholecystectomy designed around two principles: landmarking and intraoperative time-outs to determine if it is safe to progress. This standardized approach and decision tree have been helpful in promoting safe completion of laparoscopic cholecystectomies even in the most challenging cases (5). We have disseminated this approach to safe laparoscopic cholecystectomy to several divisions of General Surgery in our region through educational rounds. The purpose of this manuscript is to describe our clinical pathway and highlight the art we have developed to illustrate the algorithmic approach. These educational materials may be valuable tools for the broader community of General Surgeons to promote a culture of safety for laparoscopic cholecystectomies.

\section{Intraoperative Time-out \#1}

The first intraoperative timeout aims to set the surgeon up for safety. This involves thorough landmarking of relevant structures and assessing the need for maneuvers to optimize exposure before starting dissection. We believe a commitment to diligent intraoperative planning and setup will render even the most difficult laparoscopic cholecystectomy facile to approach with safe and reproducible results.

There are several anatomic features that should be identified in all cholecystectomies to help orient the surgeon. In our institution, these landmarks are the fundus of the gallbladder (which may be the only immediately identifiable structure), the porta hepatis, the duodenum, the sulcus of Rouviere, the cystic plate, the hilar plate, the umbilical fissure, and the inferior boundary of dissection, which we also refer to as the Line of Safety (6). Another widely used approach is the B-SAFE landmarks; the bile duct, the sulcus of Rouviere, the left hepatic artery pulsation, the umbilical fissure, and the duodenum (Enteric) (7). According to expert consensus, the most important landmark to identify prior to dissection is the sulcus of Rouviere (8). The Sulus of Rouviere is a reliable landmark present in $83 \%$ of patients undergoing laparoscopic cholecystectomy and located superior to the common bile duct in $79 \%$ of cases $(9,10)$. The Line of Safety is a theoretical line drawn through the Sulcus of Rouviere to the junction of the cystic and hilar plates (11). The sulcus of Rouviere corresponds to the right posterior portal pedicle in $70 \%$ of cases, while the junction of the cystic and hilar plate approximates to the location of the right anterior portal pedicle (12). When dissection is performed only above the Line of Safety, dangerous dissection around the

\footnotetext{
^ ORCID: 0000-0002-7161-753X.
} 
common bile duct is avoided and bile duct injury may be prevented (11). The importance of a systematic approach to landmarking, which should include the Sulcus of Rouviere, is echoed by experts who report that $76.2 \%$ of bile duct injuries can be attributed to misidentification of the local anatomy (8).

Next, the gallbladder must be securely grasped to achieve proper retraction and visualization of the cystohepatic triangle (8). With the patient positioned in reverse Trendelenburg with slight upward tilt of the right side, the lateral aspect of the gallbladder fundus is grasped and reflected towards the patient's right shoulder. Hartman's pouch is retracted towards the patient's right hip to splay out the cystohepatic triangle for dissection. In the setting of acute cholecystitis, it can be difficult to securely grasp a distended, tense gallbladder. In such circumstances, it may be necessary to decompress the gallbladder by inserting a trocar directly into the gallbladder lumen to suction its contents in a controlled fashion, thereby minimizing bile spillage and contamination.

Once adequate retraction is achieved, the cystohepatic triangle is evaluated for overlying inflammation and fibrosis. The peritoneum overlying the cystohepatic triangle has been termed the "Shield of McElmoyle" (13). Hostile, stiff, and inflamed tissue in this region should alert the surgeon to the potential for biliary injury. If it is unsafe to dissect through the Shield of McElmoyle, we recommend "toparound" mobilization of the gallbladder or opening the gallbladder for controlled removal of stones (5). The toparound approach begins with dissection above the Line of Safety, along the medial border of the gallbladder. The plane is developed in a counter-clockwise fashion between the gallbladder fundus and cystic plate. This approach, by definition, begins above the biliary pedicles and dissects away from them before stopping the dissection above the Line of Safety on the lateral side of the gallbladder. From the fundus, the plane is extended towards Hartmann's pouch until the gallbladder is separated from the gallbladder fossa. An alternative approach is to open the gallbladder and extract stones in a controlled manner. The Line of Safety may be used as a landmark above which it is safe to open the gallbladder to remove stones and visualize the cystic duct orifice from inside the gallbladder $(6,11)$.

\section{Intraoperative Time-out \#2}

The second intraoperative time-out serves to determine if the critical view of safety (CVS) has been achieved.
The CVS stipulates that the lower third of the gallbladder is dissected away from the cystic plate, the cystic triangle is cleared of fibrofatty tissues, and that two and only two structures (cystic duct and cystic artery) are visualized entering the gallbladder. These criteria are satisfied with anterior and posterior views (doublet view) $(8,11)$. If the CVS cannot be obtained due to a hostile cystohepatic triangle, the safe alternative is to pursue a subtotal cholecystectomy to prevent bile duct injury $(5,8)$.

Subtotal cholecystectomy involves removal of the distal portion of the gallbladder, in order to avoid the need for dissection of a hostile cystohepatic triangle. It is classified as fenestrating or reconstituting depending on whether the gallbladder remnant is left open or closed (14). Subtotal fenestrating cholecystectomy involves leaving a lip of proximal gallbladder tissue, containing the cystic duct orifice, open, and placing an external drain in the gallbladder fossa (14). It is associated with a higher incidence of postoperative biliary fistula, compared to the reconstituting type (14). Subtotal reconstituting cholecystectomy involves closing the proximal gallbladder remnant using endoloop, staples, or sutures, or closing the cystic duct orifice from the inside using a purse-string suture (14). This technique may be associated with reformation of gallstones and a higher recurrence of symptomatic cholelithiasis, which may require re-operation (15).

A last resort would be to convert from laparoscopic to open approach. The decision to convert is made on the basis of ensuring patient safety. Conversion to open surgery should be rare, published rates are as low as $2.6 \%$ due to worse cosmesis, longer hospital length of stay and recovery, and possibly increased post-operative complications such as pneumonia and wound infection (16-18). However, it may be the safest or best option in several situations. The most common intraoperative findings that prompt conversion to an open procedure are inflammation, adhesions, and unclear anatomy (16). Moreover, in cases where it is difficult to control bleeding, opening can be a safe and quick way of gaining control, rather than deploying excessive clips or sutures dangerously and imprecisely if the bleeding cannot be easily controlled laparoscopically. If the gallbladder is gangrenous and cannot be completely removed laparoscopically, the procedure must be converted to open to remove all necrotic tissue. Laparoscopic cholecystectomy remains the gold standard for most cases.

Clips may only be deployed once the CVS is achieved. The dissected cystic duct and artery should be clipped with $6 \mathrm{~mm}$ clips. If larger clips are required, the surgeon should 
be able to justify the reason for enlarged cystic structures, such as gallstone pancreatitis and choledocholithiasis. If the surgeon is unable to clearly identify a cause for dilation of the cystic structures, an intraoperative consult from a hepato-pancreato-biliary (HPB) surgeon or another experienced surgeon is recommended. If a HBP specialist or experienced colleague is not imminently available, a telephone or virtual consult can be sought. This serves as an additional safety check as larger clips or other methods such as staplers are commonly associated with bile duct injuries in our experience.

In addition to limiting clip size in the absence of clear indications, the number of clips used should follow "The Six Hemoclip Rule", which states that a structure in the cystohepatic triangle requires no more than three clips: two clips placed on the staying side and one clip on the specimen side (19). Therefore, a total of six clips is all that is usually needed. Expert consensus recommends against excessive, unnecessary clipping (8). If a total of more than six clips is required, exception to the rule should be justified by examining for evidence of anomalous biliary or vascular anatomy, using intraoperative cholangiography (IOC) or near-infrared fluorescent cholangiography (NIRF-C). At our institution, IOC is our navigational modality of choice as it is readily available and does not require pre-operative administration of indocyanine green dye for optimal imaging (20). If anomalous anatomy is not identified, an inperson, telephone, or virtual intraoperative consult from a HBP surgeon is again advised. After ensuring the CVS is achieved and that appropriately sized clips are intentionally placed, the surgeon can then proceed to divide the two structures between the clips, completing the laparoscopic cholecystectomy.

\section{Conclusions}

In summary, laparoscopic cholecystectomy is a common procedure that carries with it a risk of devastating complications. Following a standardized approach and decision tree can help keep patients safe from bile duct injury. Several guiding principles should be taken into account for every laparoscopic cholecystectomy. These include landmarking, consideration of gallbladder decompression, maneuvers to facilitate clearance of the cystohepatic triangle, delineation of the CVS, consideration of imaging modalities as IOC and NIRF-C, as well as bailout options including top-around approach and subtotal cholecystectomy, and conservative use of clips with respect to size, number, and placement. The algorithm outlined in this manuscript has been presented in the form of an infographic that we have developed for rapid and eyecatching knowledge dissemination. The interested reader can find this in Appendix 1. Our goal is to foster an approach to laparoscopic cholecystectomy wherein even the most difficult cases can be performed safely and confidently.

\section{Acknowledgments}

Funding: None.

\section{Footnote}

Conflicts of Interest: All authors have completed the ICMJE uniform disclosure form (available at http://dx.doi. org/10.21037/aos-21-1). SJ serves as an unpaid editorial board member of Art of Surgery. The authors have no conflicts of interest to declare.

Ethical Statement: The authors are accountable for all aspects of the work in ensuring that questions related to the accuracy or integrity of any part of the work are appropriately investigated and resolved.

Open Access Statement: This is an Open Access article distributed in accordance with the Creative Commons Attribution-NonCommercial-NoDerivs 4.0 International License (CC BY-NC-ND 4.0), which permits the noncommercial replication and distribution of the article with the strict proviso that no changes or edits are made and the original work is properly cited (including links to both the formal publication through the relevant DOI and the license). See: https://creativecommons.org/licenses/by-nc-nd/4.0/.

\section{References}

1. Elshaer M, Gravante G, Thomas K, et al. Subtotal cholecystectomy for "difficult gallbladders": systematic review and meta-analysis. JAMA Surg 2015;150:159-68.

2. Martin D, Uldry E, Demartines N, et al. Bile duct injuries after laparoscopic cholecystectomy: 11-year experience in a tertiary center. Biosci Trends 2016;10:197-201.

3. Törnqvist B, Strömberg C, Persson G, et al. Effect of intended intraoperative cholangiography and early detection of bile duct injury on survival after cholecystectomy: population based cohort study. BMJ 2012;345:e6457. 
4. Fletcher DR, Hobbs MS, Tan P, et al. Complications of cholecystectomy: risks of the laparoscopic approach and protective effects of operative cholangiography: a population-based study. Ann Surg 1999;229:449-57.

5. Purzner RH, Ho KB, Al-Sukhni E, et al. Safe laparoscopic subtotal cholecystectomy in the face of severe inflammation in the cystohepatic triangle: a retrospective review and proposed management strategy for the difficult gallbladder. Can J Surg 2019;62:402-11.

6. Greene B, Tsang M, Jayaraman S. The inferior boundary of dissection as a novel landmark for safe laparoscopic cholecystectomy. HPB (Oxford) 2021. doi: 10.1016/ j.hpb.2021.02.004.

7. Sebastian M, Sebastian A, Rudnicki J. The evaluation of B-SAFE and ultrasonographic landmarks in safe orientation during laparoscopic cholecystectomy. Wideochir Inne Tech Maloinwazyjne 2020;15:546-52.

8. Iwashita Y, Hibi T, Ohyama T, et al. Delphi consensus on bile duct injuries during laparoscopic cholecystectomy: an evolutionary cul-de-sac or the birth pangs of a new technical framework? J Hepatobiliary Pancreat Sci 2017;24:591-602.

9. Cheruiyot I, Nyaanga F, Kipkorir V, et al. The prevalence of the Rouviere's sulcus: A meta-analysis with implications for laparoscopic cholecystectomy. Clin Anat 2021;34:556-64.

10. Jha AK, Dewan R, Bhaduria K. Importance of Rouviere's sulcus in laparoscopic cholecystectomy. Ann Afr Med 2020;19:274-7.

11. Wakabayashi G, Iwashita Y, Hibi T, et al. Tokyo Guidelines 2018: surgical management of acute cholecystitis: safe steps in laparoscopic cholecystectomy for acute cholecystitis (with videos). J Hepatobiliary Pancreat Sci 2018;25:73-86.

12. Dahmane R, Morjane A, Starc A. Anatomy and surgical

doi: $10.21037 /$ aos-21-1

Cite this article as: Deng SX, Zhu A, Tsang M, Greene B, Jayaraman S. Staying safe with laparoscopic cholecystectomy: the use of landmarking and intraoperative time-outs. Art Surg 2021;5:1. relevance of Rouviere's sulcus. ScientificWorldJournal 2013;2013:254287.

13. McELMOYLE WA. Cholecystectomy: a method for the difficult gall-bladder. Lancet. 1954;266:1320-3.

14. Strasberg SM, Pucci MJ, Brunt LM, et al. Subtotal Cholecystectomy-"Fenestrating" vs "Reconstituting" Subtypes and the Prevention of Bile Duct Injury: Definition of the Optimal Procedure in Difficult Operative Conditions. J Am Coll Surg 2016;222:89-96.

15. Bodvall B, Overgaard B. Cystic duct remnant after cholecystectomy: incidence studied by cholegraphy in 500 cases, and significance in 103 reoperations. Ann Surg 1966;163:382-90.

16. Le VH, Smith DE, Johnson BL. Conversion of laparoscopic to open cholecystectomy in the current era of laparoscopic surgery. Am Surg 2012;78:1392-5.

17. Coccolini F, Catena F, Pisano M, et al. Open versus laparoscopic cholecystectomy in acute cholecystitis. Systematic review and meta-analysis. Int J Surg 2015;18:196-204.

18. Keus F, de Jong JA, Gooszen HG, et al. Laparoscopic versus open cholecystectomy for patients with symptomatic cholecystolithiasis. Cochrane Database Syst Rev 2006;(4):CD006231.

19. Wall WJ, Passi RB. Laparoscopic cholecystectomy: the six hemoclip rule. Can J Surg 1992;35:575.

20. Tsutsui N, Yoshida M, Nakagawa H, et al. Optimal timing of preoperative indocyanine green administration for fluorescent cholangiography during laparoscopic cholecystectomy using the PINPOINT® Endoscopic Fluorescence Imaging System. Asian J Endosc Surg 2018;11:199-205. 


\section{Safe Laparoscopic Cholecystectomy}

\section{TIME-OUT \#1: Landmarking and Operative Planning}

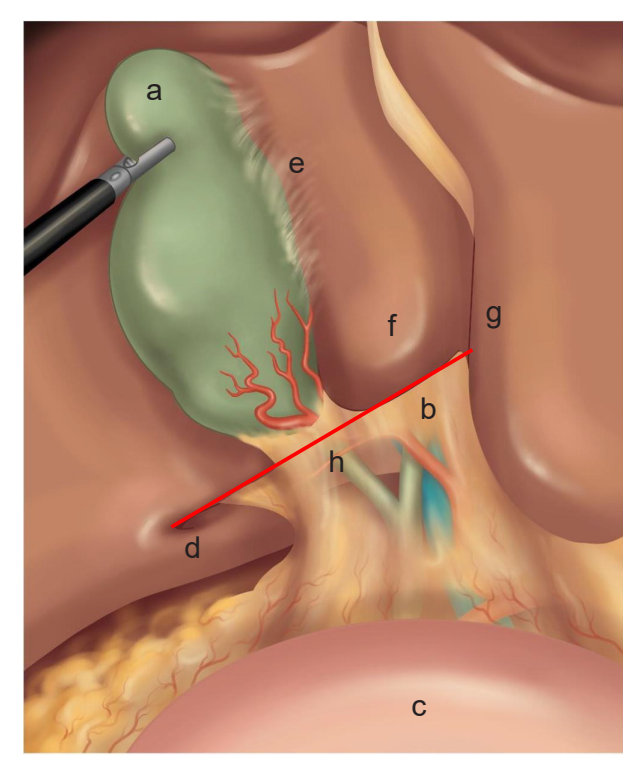

\section{TIME-OUT \#2: Is it Safe to Fire Clips?}

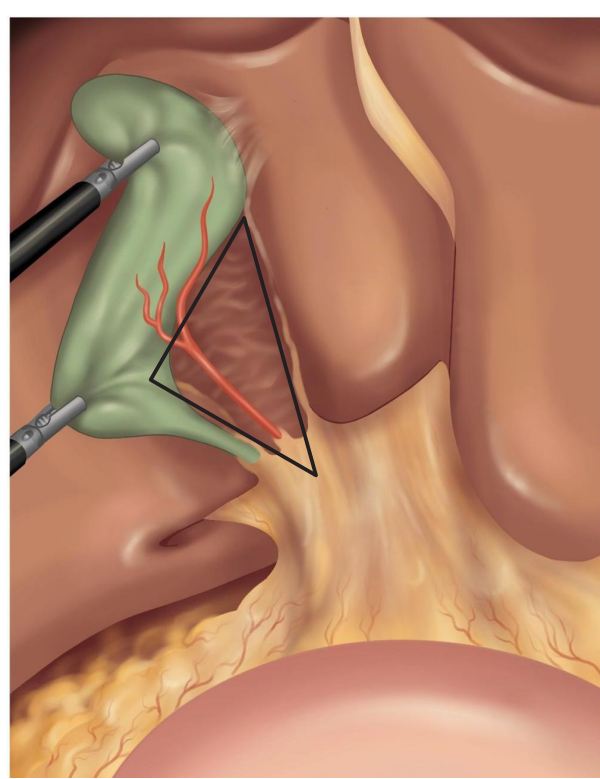

STEP 1: Landmark

a. Gallbladder fundus,

b. Porta hepatis

c. Duodenum

d. Sulcus of Rouviere

e. Cystic plate

f. Hilar plate

g. Umbilical fissure

h. LINE OF SAFETY

\section{STEP 2a: Safety Measures}

Does the gallbladder need decompression to be grasped?

$$
\text { No } \downarrow \text { Yes }
$$

STEP 2b: Safety Measures

Is the cystohepatic triangle hostile (Shield of McElmoyle)?
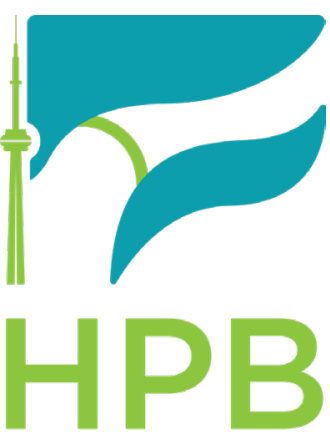

S T. J O E, S

Decompress gallbladder

Consider maneuvers

above LINE OF

SAFETY:

- Top-around mobilization of gallbladder fossa

- Opening gallbladder for controlled removal of stones Has critical view of safety been achieved?

- Lower $1 / 3$ gallbladder dissected from cystic plate

- Cystohepatic triangle cleared

- Two and only two structures entering gallbladder

- Doublet view Yes $\downarrow$ No

cholecystectomy:

- Reconstituting

- Fenestrating

\section{STEP 4: Clip Size}

Am I only using $6 \mathrm{~mm}$ clips?

$$
\text { Yes } \leftarrow \text { No }
$$

STEP 5: Number of Clips

Am I using more than six clips?

$$
\text { No } \downarrow \text { Yes }
$$

STEP 6: Clip Placement

Place one clip on specimen side and two clips on staying side

\section{Clearly justify (gallstone pancreatitis, \\ choledocholithiasis), \\ consider IOC, NIRF-C or intraoperative consult \\ Clearly justify \\ (anatomical anomaly). consider IOC, NIRF-C or intraoperative consult IOC (intraoperative cholangiography)}

*INDICATIONS FOR: Conversion to Open Approach

Extensive adhesions, unclear anatomy, difficulty controlling bleeding (do not fire clips blindly or without clear anatomy), gangrenous gallbladder that cannot be completely removed laparoscopically 